\title{
Atom-optics hologram in the time domain
}

\author{
A. V. Soroko* \\ National Centre of Particle and High Energy Physics, Belarusian State University, \\ Bogdanovich Street 153, Minsk 220040, Belarus
}

\begin{abstract}
The temporal evolution of an atomic wave packet interacting with object and reference electromagnetic waves is investigated beyond the weak perturbation of the initial state. It is shown that the diffraction of an ultracold atomic beam by the inhomogeneous laser field can be interpreted as if the beam passes through a three-dimensional hologram, whose thickness is proportional to the interaction time. It is found that the diffraction efficiency of such a hologram may reach $100 \%$ and is determined by the duration of laser pulses. On this basis a method for reconstruction of the object image with matter waves is offered.
\end{abstract}

03.75.Be, 42.50.Vk, 32.80.Lg, 81.15.Fg

\section{INTRODUCTION}

The success of the last decade in the field of laser light cooling below the recoil limit [1.2] has opened a new chapter of atom optics, whose objective is to manipulate atomic beams in a way similar to conventional optics by exploiting the wave properties of the particles. Indeed, if the momenta of cooled atoms approach those of photons, diffraction effects may manifest themselves particularly strongly during atomic interaction with spatially inhomogeneous radiation. For the corresponding part of the de Broglie wave spectrum, this provides the possibility of supplementing the traditional atom-optics set of elements such as mirrors [3], diffraction gratings [4.5], or lenses [6] with holograms of different objects, the conventional optics analogs of which have been well known for several decades [7]. Creation of matter waves with the required amplitude and phase characteristics is the main task assigned to such atomic holograms. Since these characteristics are the same as those of the object wave, one obtains a powerful and convenient tool for holographic imaging with atoms. The latter may have useful practical applications ranging from atom lithography [8] to the manufacturing of microstructures or quantum microfabrication.

One possibility to make an atomic hologram is to create a mechanical mask with appropriate transparency for the incident atomic beam (the analog of a twodimensional optical hologram). Such a hologram has the advantage of being permanent. However, up to now only masks with binary transparency have been prepared. For example, in the experiment 9 the mask was written onto a thin silicon nitride membrane and allowed for either complete or vanishing transmission of the beam at a given point. Since gradually varying transmission of the beam is required for the correct holographic storage of information, this reduces resolution in the reconstructed image.

A very interesting proposal has recently been reported in Ref. [10], where the authors regard a Bose-Einstein condensate (BEC) as the registration medium for the atomic hologram. In the suggested method the information issuing from an object is encoded into the condensate in the form of density modulations by using the object and reference laser beams which form the writing optical potential. The reconstruction of matter wave arises due to s-wave scattering of the reading-beam atoms on condensate inhomogeneities. This proposal illustrates the wide potential applicability of the BEC, which, since it was realized experimentally [11], is now available almost routinely in several laboratories.

In a previous paper 12] we have shown that an atomic hologram may also be constructed under certain conditions as a superposition of reference and object electromagnetic waves, which is common for optical holography. The creation of the intended matter wave occurs when an ultracold atomic beam is diffracted from this hologram, which in fact is just an inhomogeneous light field. The main advantages of the proposed scheme are its simplicity because of bypassing the recording process and, as a consequence, the absence of aberrations in the stored information. In this sense our approach is close to the non holographic scheme of wave front engineering [13, in which the center-of-mass wave function of an atom can be arbitrarily shaped by means of a sequence of suitably formed laser pulses.

The main assumption employed in our holographic scheme is the linear response of the atomic system to the laser-field inhomogeneity. It requires, in particular, that the perturbation of the incident atomic beam is weak, and sets an upper limit on the object wave amplitude [see Eq. (50) in Ref. [12]]. As a result, only a small portion of atoms in the beam can be transferred into the reconstructed matter wave. Operating in the linear-response regime decreases the diffraction efficiency of an atomic hologram, i.e., the ratio of the intensity of diffracted atomic waves to the intensity of the reading beam, which may be crucial for practical applications. In conventional optical holography such a situation corresponds to the kinematical regime of information recording [7]. On the other hand, the coupled wave theory of Kogelnik [14], and the theories based on the dynamical approximation [15 177, provide a recipe for creating a hologram with high (up to 100\%) diffraction efficiency. To achieve this goal it is necessary to control, among other parameters, 


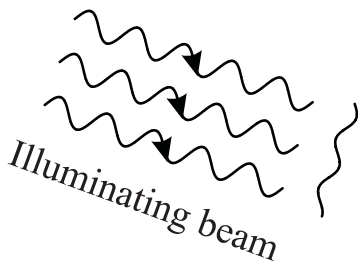

$\hat{\gamma}$ Object
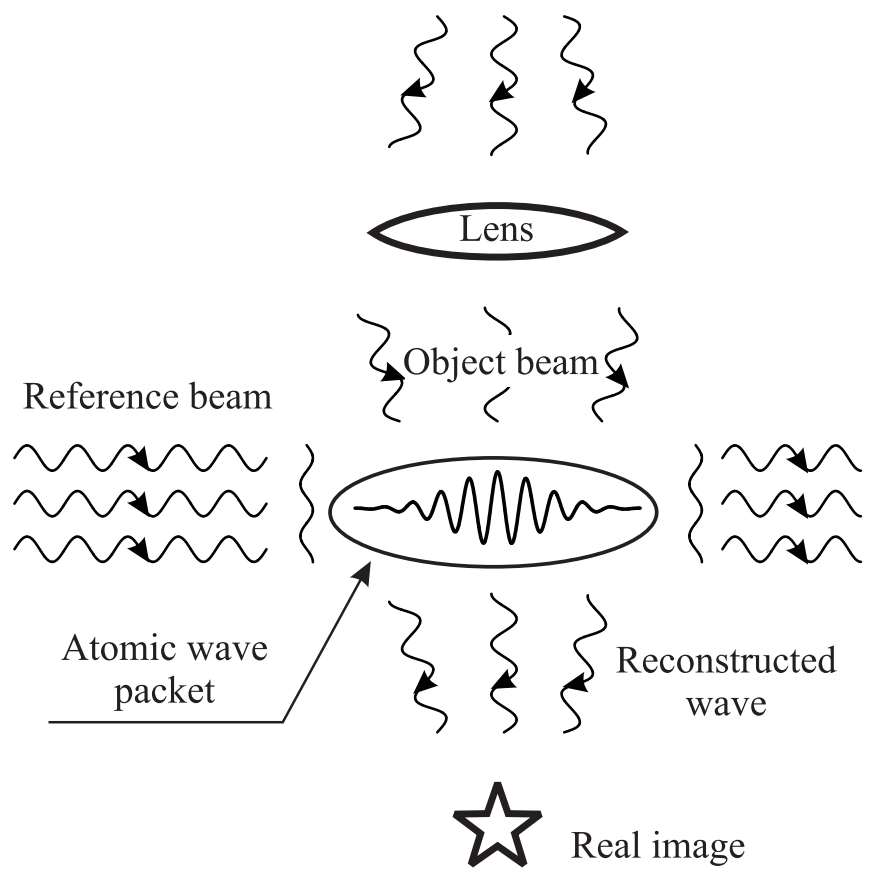

FIG. 1. Typical layout design of laser beams and matter wave packets suitable for atomic holography.

the thickness of the hologram. Unfortunately, thickness controlling is difficult in a scheme of atom holography without a registration medium like the one in Ref. [12] (see Fig. 1 for details).

The purpose of the present paper is to suggest an approach for creation of atom optics holograms that will combine the advantages of our previous scheme with high diffraction efficiencies. We will show that high diffraction efficiencies can be realized if one restricts the extent of the atomic hologram in the time domain rather than in space. The hologram will thus work in a pulsed regime pumping atoms from the beam or from the initial wave packet into the reconstructed wave. Note that the suggested regime is well compatible with Raman cooling methods [2] (including laser cooling below the gravitational limit [18]) and the recent realization of an atom laser |19], which are capable of repeatedly reproducing the coherent or almost coherent atomic wave packets necessary for actual implementation of a reading beam.

Another important prerequisite for successful wave front reconstruction with massive particles concerns the need to compensate for the potentially detrimental influence of gravitational effects. Fortunately, the bulk of the atoms possess a magnetic moment, and all one has to do is use the Stern-Gerlach effect. Superimposing the weakly inhomogeneous magnetic field onto the path of prepolarized particles and appropriately adjusting the field gradient, it is possible to suspend the ground-state atoms everywhere except in the region of interaction with radiation. But if the laser frequency is far from all atomic transitions, the contribution to the total force induced by spatially dependent shifts of the Zeeman levels is negligible. Under this condition, atoms move like free particles that are affected only by the electromagnetic waves.

In Sec. II we specify the magnetic field to compensate for the gravity effects and derive an equation that describes the dynamics of ground-state atoms interacting with the object and reference beams. An approximate solution of this equation is found without assuming linear response to laser-field inhomogeneity, and its domain of validity is determined. For reasonable experimental conditions the solution admits an atom-optics interpretation, which is presented in Sec. III, namely, the inhomogeneous laser radiation is shown to behave like a threedimensional hologram with respect to the impinging wave packets. A numerical simulation of such a hologram created with a 31-mode object beam is carried out, and high diffraction efficiency is explicitly demonstrated. Section IV concludes with a summary of the results. Certain mathematical details concerning derivation of the basic formulas are relegated to the Appendixes.

\section{BASIC FORMULAS}

\section{A. Compensation for gravity}

To be specific, let us consider an atom with a $J=\frac{1}{2}$ to $J=\frac{3}{2}$ transition, e.g., sodium or cesium. A magnetic field $\mathbf{B}(\mathbf{r})$ is applied to compensate for gravity. It is supposed to contain a homogeneous component $\mathbf{B}_{0}$ directed along the gravity acceleration $\mathbf{B}_{0} \uparrow \uparrow \mathbf{g}$. The remaining inhomogeneous part of the field $\mathbf{B}_{1}(\mathbf{r})=\mathbf{B}(\mathbf{r})-\mathbf{B}_{0}$ should be small compared to this component,

$$
\left|\mathbf{B}_{1}(\mathbf{r})\right| \ll B_{0}=\left|\mathbf{B}_{0}\right| .
$$

As we will see below, to fulfil this condition it is necessary to take $B_{0}$ in the range $10^{3}-10^{4} \mathrm{G}$. In practice such a field is strong enough to induce Zeeman shifts which considerably exceed the hyperfine splitting intervals $\sim \hbar \omega_{\text {HFS }}$ (but not the multiplet ones). Therefore an internal atomic eigenstate $\left|J, I, M_{J}, m_{I}\right\rangle$ may be parametrized by the set of quantum numbers consisting of the angular momenta of the electronic shell $J$ and nucleus $I$, and their local projections onto the direction of the magnetic field, $M_{J}$ and $m_{I}$. The corresponding energy eigenvalue is determined not only by the multiplet level $E_{J}$ but also by the magnetic field $B(\mathbf{r})=|\mathbf{B}(\mathbf{r})|$ and therefore is spatially dependent,

$$
\begin{aligned}
E_{\left|J, I, M_{J}, m_{I}\right\rangle}(\mathbf{r})= & E_{J}+a M_{J} m_{I} \\
& +\left(\mu_{B} g_{L} M_{J}-\mu_{\mathrm{nuc}} m_{I}\right) B(\mathbf{r}),
\end{aligned}
$$


where $a$ is the hyperfine coupling constant $\left(a \propto \hbar \omega_{\mathrm{HFS}}\right.$, e.g., for $\mathrm{Na} a / \hbar=885.8 \mathrm{MHz}), g_{L}$ denotes the Landé factor, and $\mu_{\text {nuc }}$ is the nuclear magnetic moment. Because of the condition (2.1), such a spatial dependence, however, mainly arises from the longitudinal $\left[B_{1}^{\|}(\mathbf{r})=\right.$ $\left.\mathbf{B}_{0} \cdot \mathbf{B}_{1}(\mathbf{r}) / B_{0}\right]$, rather than the transverse $\left[\mathbf{B}_{1}^{\perp}(\mathbf{r})\right]$ component of the vector $\mathbf{B}_{1}(\mathbf{r})$, provided that the components are defined relative to $\mathbf{B}_{0}$. This is evident from the expression

$$
\begin{aligned}
B(\mathbf{r})= & \sqrt{\left[B_{0}+B_{1}^{\|}(\mathbf{r})\right]^{2}+\left[\mathbf{B}_{1}^{\perp}(\mathbf{r})\right]^{2}} \\
& \simeq B_{0}+B_{1}^{\|}(\mathbf{r})+\left[\mathbf{B}_{1}^{\perp}(\mathbf{r})\right]^{2} /\left(2 B_{0}\right)
\end{aligned}
$$

where the term containing $\mathbf{B}_{1}^{\perp}(\mathbf{r})$ is small and can be neglected. Consequently, by adjusting the gradient of the field $B_{1}^{\|}(\mathbf{r})$ one can achieve translation invariance of the ground state $|g\rangle=|1 / 2, I,-1 / 2, I\rangle$ (or another state with $J=1 / 2$ ) in three dimensions:

$$
E_{|g\rangle}(\mathbf{r})-M \mathbf{g} \cdot \mathbf{r}=\text { const. }
$$

For example, to balance the gravitational force in this way for sodium it is necessary to create a gradient $\nabla B_{1}^{\|}(\mathbf{r})=b_{1} \mathbf{g} /|\mathbf{g}|$, where $b_{1}=-4.033 \mathrm{G} / \mathrm{cm}$. This condition does not contradict the Maxwell equation $\nabla$. $\mathbf{B}_{1}(\mathbf{r})=0$, because variation of $\mathbf{B}_{1}^{\perp}(\mathbf{r})$ is not restricted. Note also that the choice $B_{0}=10^{3}-10^{4} \mathrm{G}$ maintains condition (2.1) very well within a spatial region of size $\sim 10 \mathrm{~cm}$.

All the other levels are affected by the residual external potential. In particular, the force $\mathbf{f}_{e}$ acting on the atoms in the excited state, e.g., $|e\rangle=|3 / 2, I,-3 / 2, I\rangle$, may be estimated from Eqs. (2.2) and (2.4) as $\left|\mathbf{f}_{e}\right| \sim M g$.

\section{B. Dynamics of the ground-state atoms}

In our scheme, we use pulses of laser light at frequency $\omega$ which is roughly tuned to the $|g\rangle \rightarrow|e\rangle$ transition. If the typical size $2 L$ of the atomic sample is restricted by the condition $L \ll a /(M g)$, one may regard $E_{|e\rangle}(\mathbf{r})$ as the excited level that is closest to resonance within the whole interaction domain. Indeed, the maximal spatial shift of the level $\sim M g L$ induced by the force $\mathbf{f}_{e}$ appears to be much less than the hyperfine splitting intervals $\left(M g L \ll a \sim \hbar \omega_{\mathrm{HFS}}\right)$, and the hierarchy of detunings is retained. Therefore an atom initially in the $|g\rangle$ state behaves as a two-level system with respect to the processes with stimulated emission of photons.

The atom moves inside a superposition of the reference and the object beams during the whole laser pulse. Each beam is represented as a discrete sum of plane monochromatic electromagnetic waves. In particular, we use the following decomposition of the electric field in the object beam:

$$
\mathbf{E}_{s}(\mathbf{r}, t)=\sum_{m \geq 1} \mathbf{E}_{m} \exp \left(i \mathbf{k}_{m} \cdot \mathbf{r}-i \omega t\right)+\text { c.c. },
$$

where $\mathbf{E}_{m}$ and $\mathbf{k}_{m}$ stand for the complex amplitude of the mode $m$ and its wave vector, respectively. Such an approach does not restrict the generality of our consideration, because the expression (2.5) must well describe the real laser field only in the atom-laser interaction region. Evidently, the latter requirement can always be satisfied by decreasing the minimal angle between the mode wave vectors. In this case we can also regard the reference beam as a single mode (with the index $m=0$ ),

$$
\mathbf{E}_{r}(\mathbf{r}, t)=\mathbf{E}_{0} \exp \left(i \mathbf{k}_{0} \cdot \mathbf{r}-i \omega t\right)+\text { c.c. },
$$

which is a typical arrangement for optical holography.

Since the atomic dipole momentum operator $\hat{\mathbf{d}}$ is diagonal in quantum numbers $I$ and $m_{I}$, the transitions that change $m_{I}$ are allowed only due to hyperfine interaction. As a consequence, the excited state $|e\rangle$ decays to the lower ones preferentially in the channel $|e\rangle \rightarrow|g\rangle$ (with the rate $\gamma$ ). This circumstance makes it possible to deal with an atom as a two-level system even if spontaneous photon emission takes place. However, to simplify the consideration, the coherent scattering processes are assumed to dominate the spontaneous emission, i.e., the regime $|\Delta| \gg \gamma$ is maintained [20,21, where $\Delta=\omega+\left[E_{|g\rangle}(0)-E_{|e\rangle}(0)\right] / \hbar$ is the detuning from resonance in the center of the atom-laser interaction region $(\mathbf{r}=0)$. Under such a condition the one-particle density matrix in the momentum representation [22] has an obvious time evolution,

$$
\begin{aligned}
\rho_{a b}\left(\mathbf{p}_{1}, \mathbf{p}_{2}, t\right)= & \int d \mathbf{p}_{1}^{\prime} \int d \mathbf{p}_{2}^{\prime} \sum_{a^{\prime} b^{\prime}} G_{a a^{\prime}}\left(\mathbf{p}_{1}, \mathbf{p}_{1}^{\prime}, t\right) \\
& \times G_{b b^{\prime}}^{*}\left(\mathbf{p}_{2}, \mathbf{p}_{2}^{\prime}, t\right) \rho_{a^{\prime} b^{\prime}}\left(\mathbf{p}_{1}^{\prime}, \mathbf{p}_{2}^{\prime}, t=0\right) .
\end{aligned}
$$

Here indices $a, b \ldots$ span the internal atomic states $(e, g)$, and $G_{a a^{\prime}}\left(\mathbf{p}_{1}, \mathbf{p}_{1}^{\prime}, t\right)$ is the Green function of the twocomponent Schrödinger equation describing atomic dynamics during the $|g\rangle \leftrightarrow|e\rangle$ transitions [see Eq. (A1) for the details].

For the situation at hand, the upper electronic state can be adiabatically eliminated from consideration (see Appendix A provided that the detuning $\Delta$ is large enough [5,20],

$$
|\Delta| \gg\left|\Omega_{m}\right|,\left|\mathbf{f}_{e}\right| L / \hbar,
$$

where $\Omega_{m}=\left\langle e\left|\hat{\mathbf{d}} \cdot \mathbf{E}_{m}\right| g\right\rangle / \hbar$ is the Rabi frequency of mode $m$. As a result, the dynamics of the ground atomic state is completely determined by the equation for the centerof-mass wave function $\psi_{g}(\mathbf{p}, t)$,

$$
\begin{aligned}
i \frac{\partial}{\partial t} \psi_{g}(\mathbf{p}, t)= & {\left[w(\mathbf{p})+\Delta+f_{0}\right] \psi_{g}(\mathbf{p}, t) } \\
& +\sum_{m \geq 1}\left(\sum_{\substack{n \geq 1 \\
n \neq m}} f_{m n} \psi_{g}\left[\mathbf{p}-\hbar\left(\mathbf{k}_{m}-\mathbf{k}_{n}\right), t\right]\right.
\end{aligned}
$$




$$
\begin{aligned}
& +g_{m} \psi_{g}\left[\mathbf{p}-\hbar\left(\mathbf{k}_{m}-\mathbf{k}_{0}\right), t\right] \\
& \left.+g_{m}^{*} \psi_{g}\left[\mathbf{p}+\hbar\left(\mathbf{k}_{m}-\mathbf{k}_{0}\right), t\right]\right),
\end{aligned}
$$

where $w(\mathbf{p})$ denotes the kinetic energy (in units of $\hbar$ ), and $f_{0}, f_{m n}$, and $g_{m}$ stand for the effective Rabi frequencies, introduced by Eqs. (A3).

\section{Evolution of wave packets}

It is known from the theory of thick optical holograms that reconstruction of the original (conjugate) object wave arises only if the reading beam is directed along (opposite) the reference wave and has the same wavelength. In analogy with conventional optics, let us consider for definiteness the evolution of an atomic wave packet whose spectrum is initially concentrated around the mean momentum of photons in the reference beam. In such a case one can expect creation of a matter wave similar to the forward object wave. Therefore it is convenient to seek the solution of Eq. (2.9) as a sum of wave packets approaching the plane modes of the hologram [16],

$$
\psi_{g}(\mathbf{p}, t)=\sum_{m \geq 0} \psi_{m}\left(\mathbf{p}-\hbar \mathbf{k}_{m}, t\right)
$$

Initially there are no wave packets corresponding to the object beam, so that

$$
\psi_{m}(\mathbf{p}, t=0)=0, \quad m \geq 1
$$

and as a consequence,

$$
\psi_{0}\left(\mathbf{p}-\hbar \mathbf{k}_{0}, t=0\right)=\psi_{g}(\mathbf{p}, t=0) \equiv \psi_{g}(\mathbf{p}) .
$$

Population of these atomic motional states $(m \geq 1)$ arises due to coupling with $\psi_{0}(\mathbf{p}, t)$, the wave packet corresponding to the reference beam,

$$
i \frac{\partial}{\partial t} \psi_{m}(\mathbf{p}, t)=w_{m}(\mathbf{p}) \psi_{m}(\mathbf{p}, t)+g_{m} \psi_{0}(\mathbf{p}, t),
$$

where

$$
w_{m}(\mathbf{p})=w\left(\mathbf{p}+\hbar \mathbf{k}_{m}\right)+\Delta+f_{0} .
$$

Depletion of the state with $m=0$ is governed by the equation

$i \frac{\partial}{\partial t} \psi_{0}(\mathbf{p}, t)=w_{0}(\mathbf{p}) \psi_{0}(\mathbf{p}, t)+\sum_{m \geq 1} g_{m}^{*} \psi_{m}(\mathbf{p}, t)+\chi(\mathbf{p}, t)$,

which one can obtain after substituting Eqs. (2.10) and (2.13) into Eq. (2.9).

Equation (2.9) is thus reduced to the system of equations (2.13), 2.15). The advantage of this step becomes obvious after making the following self-consistent assumption about the momentum spectrum of $\psi_{m}(\mathbf{p}, t)$, $m \geq 0$, the validity of which was verified for the twomode case in Ref. [18]. We will suppose below that all nonvanishing functions have narrow distributions around $\mathbf{p}=0$ and, as a result, do not overlap in the expression for $\chi(\mathbf{p}, t)$,

$$
\begin{aligned}
\chi(\mathbf{p}, t)= & \sum_{m \geq 1}\left(\sum_{n \geq 1} g_{n} \psi_{m}\left[\mathbf{p}+\hbar\left(2 \mathbf{k}_{0}-\mathbf{k}_{n}-\mathbf{k}_{m}\right), t\right]\right. \\
& +\sum_{\substack{n \geq 0 \\
n \neq m}} g_{m}^{*} \psi_{n}\left[\mathbf{p}+\hbar\left(\mathbf{k}_{m}-\mathbf{k}_{n}\right), t\right] \\
& +\sum_{\substack{n \geq 1 \\
n \neq m}} \sum_{l \geq 0} f_{m n} \\
& \left.\times \psi_{l}\left[\mathbf{p}+\hbar\left(\mathbf{k}_{0}-\mathbf{k}_{l}-\mathbf{k}_{m}+\mathbf{k}_{n}\right), t\right]\right) .
\end{aligned}
$$

Under these conditions different parts of $\chi(\mathbf{p}, t)$ give incoherent contributions, which are small at low $g_{m}$ and can be taken into account perturbatively. In zeroorder approximation one omits $\chi(\mathbf{p}, t)$ so that the system (2.13), 2.15) becomes homomorphic with the rate equations describing an $(m+1)$-level atom. Note that the stationary solutions of this truncated system exactly coincide with eigenmodes of the corresponding optical hologram 16].

To make further progress it is convenient to perform the Laplace transformation $(m \geq 0)$,

$$
\psi_{m}(\mathbf{p}, \lambda)=\int_{0}^{\infty} d t e^{-\lambda t} \psi_{m}(\mathbf{p}, t)
$$

with the initial conditions (2.11) and (2.12). Then the equations for the Laplace transforms will allow an easy zero-order solution,

$$
\begin{gathered}
\psi_{0}^{(0)}(\mathbf{p}, \lambda)=\frac{-i}{T(\mathbf{p}, \lambda)} \psi_{g}\left(\mathbf{p}+\hbar \mathbf{k}_{0}\right), \\
\psi_{m}^{(0)}(\mathbf{p}, \lambda)=\frac{-g_{m}}{w_{m}(\mathbf{p})-i \lambda} \psi_{0}^{(0)}(\mathbf{p}, \lambda), \quad m \geq 1,
\end{gathered}
$$

where

$$
T(\mathbf{p}, \lambda)=w_{0}(\mathbf{p})-i \lambda+\sum_{m \geq 1} \frac{-\left|g_{m}\right|^{2}}{w_{m}(\mathbf{p})-i \lambda} .
$$

Similarly, the next iteration reproduces the first-order solution,

$$
\begin{gathered}
\psi_{0}^{(1)}(\mathbf{p}, \lambda)=\psi_{0}^{(0)}(\mathbf{p}, \lambda)+\frac{-\chi^{(0)}(\mathbf{p}, \lambda)}{T(\mathbf{p}, \lambda)}, \\
\psi_{m}^{(1)}(\mathbf{p}, \lambda)=\frac{-g_{m}}{w_{m}(\mathbf{p})-i \lambda} \psi_{0}^{(1)}(\mathbf{p}, \lambda), \quad m \geq 1,
\end{gathered}
$$




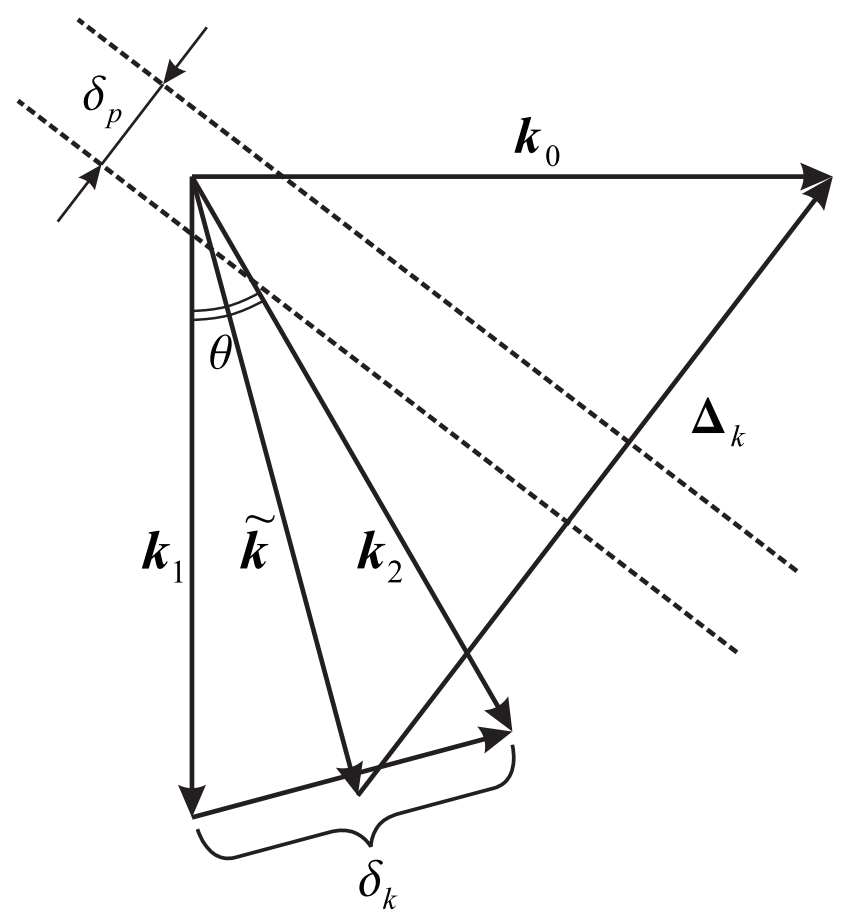

FIG. 2. Definitions of the values $\boldsymbol{\Delta}_{k}, \delta_{k}$, and $\delta_{p}$ in the simplest case of a two-mode object wave.

where $\chi^{(0)}(\mathbf{p}, \lambda)$ is obtained from the expression (2.16) after making the substitutions $\psi_{m}(\mathbf{p}, t) \rightarrow \psi_{m}^{(0)}(\mathbf{p}, \lambda)$, $m \geq 0$. In principle, we can get the solution with any desired accuracy by repeating the iterations but it will be sufficient to restrict ourselves to the first-order formulas in what follows.

The time-dependent wave functions are obtained as the inverse Laplace transforms of $\psi_{m}(\mathbf{p}, \lambda)$ according to the Mellin formula

$$
\psi_{m}(\mathbf{p}, t)=2 \pi i \int_{\epsilon-i \infty}^{\epsilon+i \infty} d \lambda e^{\lambda t} \psi_{m}(\mathbf{p}, \lambda), \quad \epsilon>0 .
$$

Finally, using Eqs. 2.20) and (2.21) one can easily obtain the ground-state component $G_{g g}\left(\mathbf{p}, \mathbf{p}^{\prime}, t\right)$ of the Green function appearing in the formula (2.7). The corresponding expression is derived in Appendix B.

\section{Validity of assumptions}

Let us first check that the zero-order solution 2.18) indeed has a narrow momentum spectrum around $\mathbf{p}=0$, provided the initial conditions are chosen properly and the effective Rabi frequency $g_{m}$ is small enough. We may restrict ourselves to examination of the region $\mathcal{D}=$ $\left\{\mathbf{p}:\left[w_{m}(\mathbf{p})-w_{0}(\mathbf{p})\right]^{2} \lesssim\left|g_{m}\right|^{2}, \forall m\right\}$, where all the functions in the truncated system of equations (2.13),(2.15) can come into resonance. In this region the equations admit an approximate analytical solution presented in Appendix C.

The main feature of the near-resonance solution (C1) is that the initial atomic wave packet transforms into motional states with $m \geq 1$ at a time $\tau_{n}$ (time of the $n \pi$ pulse [21])

$$
\tau_{n}=\frac{\pi}{2 g_{\Sigma}}(2 n-1), \quad n \in \mathcal{N} .
$$

Here $g_{\Sigma}$ stands for the overall effective Rabi frequency defined in Eq. (C6). This transition is velocity selective and is most efficient when the Bragg resonance condition $\mathbf{p} \cdot \boldsymbol{\Delta}_{k}=0$ is satisfied, where $\boldsymbol{\Delta}_{k}$ denotes the typical difference between wave vectors in the object and the reference beams (cf. Ref. 20]). The width of the peak in the momentum distribution along the direction of vector $\boldsymbol{\Delta}_{k}$ (the interval from the maximum to the first minimum) depends on the interaction time, and for $t \leq 2 \tau_{1}$ is

$$
\delta_{p}(t)=\frac{2 M g_{\Sigma}}{\Delta_{k}} \sqrt{4\left(\frac{\tau_{1}}{t}\right)-1} .
$$

For a given value $\Delta_{k}=\left|\boldsymbol{\Delta}_{k}\right|$ it decreases with $g_{\Sigma}$. Therefore the smaller the effective Rabi frequencies $g_{m}$ the narrower the momentum spectrum of $\psi_{m}^{(0)}(\mathbf{p}, t)$.

To prevent all nonvanishing functions comprising the term $\chi(\mathbf{p}, t)$ from overlapping in momentum space their spectra must be concentrated within the domain $|\mathbf{p}|<$ $\hbar \delta_{k}$ at $t \sim \tau_{1}$, where

$$
\delta_{k}=\min _{m, n \geq 0}\left|\mathbf{k}_{m}-\mathbf{k}_{n}\right|
$$

is the minimal distance between different wave vectors of the laser beams (see Fig. 2). Since the spectral extent along the direction of vector $\boldsymbol{\Delta}_{k}$ is characterized by $\delta_{p}(t)$, we immediately get the condition

$$
\delta_{p}\left(\tau_{1}\right) \ll \hbar \delta_{k} .
$$

In agreement with Eq. (2.23) it sets an upper limit on the overall effective Rabi frequency,

$$
g_{\Sigma} \ll \hbar \delta_{k} \Delta_{k} /(2 \sqrt{3} M) .
$$

In the transverse direction the spectra are the same as that of the initial wave packet $\psi_{g}\left(\mathbf{p}+\hbar \mathbf{k}_{0}\right)$. Therefore one must impose another condition,

$$
\left|\left(\mathbf{p}^{\prime}-\hbar \mathbf{k}_{0}\right) \times \boldsymbol{\Delta}_{k}\right|<\hbar \delta_{k} \Delta_{k},
$$

which restricts allowed values of $\mathbf{p}^{\prime}$ in the domain of the Green function $G_{g g}\left(\mathbf{p}, \mathbf{p}^{\prime}, t\right)$.

When the inequalities (2.25) and 2.27) are satisfied, the main correction to the zero-order solution $\psi_{m}^{(0)}(\mathbf{p}, t)$ caused by the term $\chi(\mathbf{p}, t)$ arises outside the nearresonance region $\mathcal{D}$ and depends on the geometry of laser beams. So for $t \sim \tau_{1}$ and a two-dimensional (2D) holographic setup like that in Fig. 2 (i.e., all $\mathbf{k}_{m}$ are coplanar 

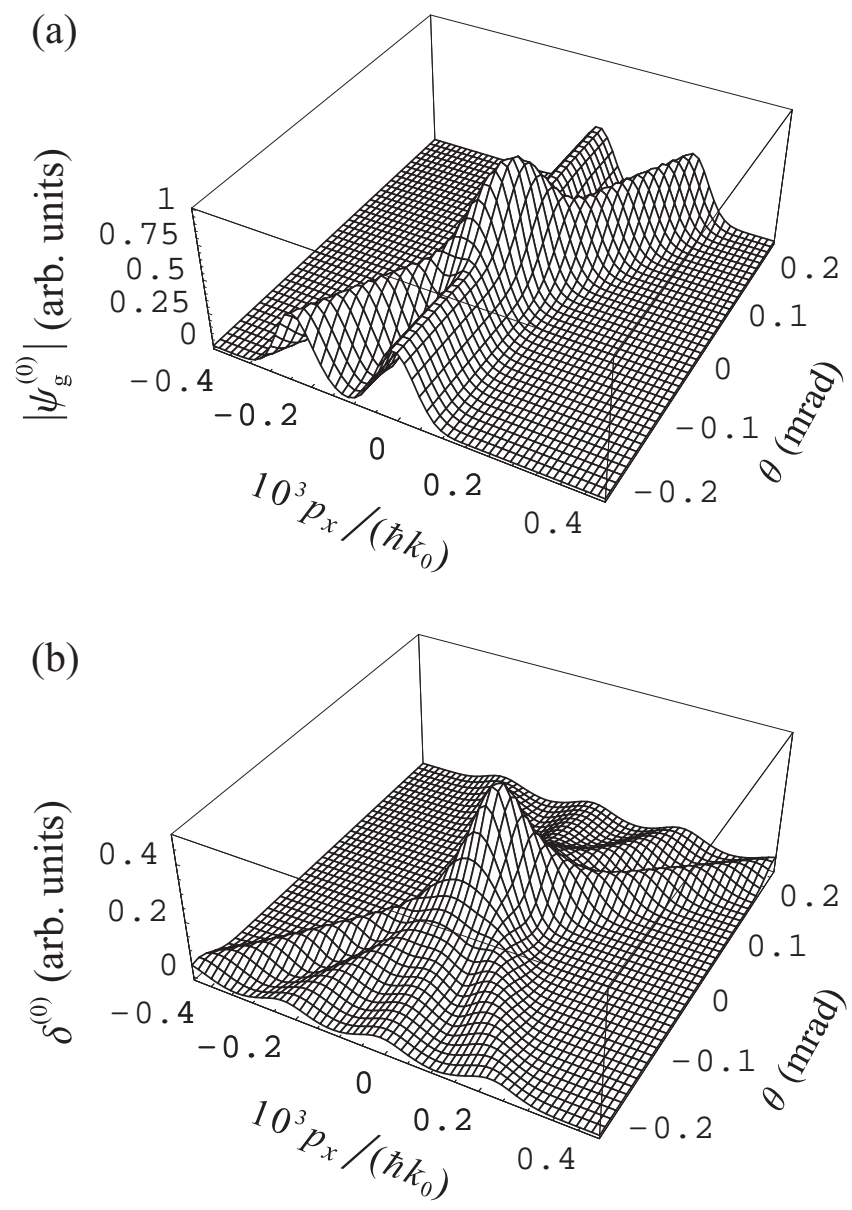

FIG. 3. Absolute values of zero-order solution $\left|\psi_{g}^{(0)}\right|$ (a) and first-order correction to it $\delta^{(0)}$ (b) as functions of the momentum component $p_{x}$ and the angle $\theta$ between $\mathbf{k}_{1}$ and $\mathbf{k}_{2}$. The arbitrary units scale is the same in both plots. The remaining components of $\mathbf{p}$ are fixed as follows: $p_{y}=\hbar k_{0}$ and $p_{z}=0$. The geometry of the laser beams is chosen as in Fig. 2. The effective Rabi frequencies $g_{1}=g_{2}=10 \mathrm{~Hz}, f_{12}=0.1$ $\mathrm{Hz}$.

vectors) the relative correction has the order of magnitude $\varepsilon_{r}=\delta_{p}\left(\tau_{1}\right) /\left(\hbar \delta_{k}\right) \ll 1$, as follows from Eqs. 2.16) and $(\overrightarrow{\mathrm{C} 1 \mathrm{~b}})$. To obtain this estimate one must apply the standard holographic restriction on the intensities of laser beams $\left|\mathbf{E}_{0}\right|^{2} \gg\left|\mathbf{E}_{m}\right|^{2}, m \geq 1$, which leads to the inequality $\left|g_{m}\right| \gg\left|f_{m n}\right|$. Then the third term in the expression (2.16) should be discarded, because it is proportional to $f_{m n}$ and, consequently, is much less than the first and second ones $\left(\propto\left|g_{m}\right|\right)$.

To illustrate the consistency of our approach in the case of a two-mode object wave let us consider absolute values of the zero-order solution $\left|\psi_{g}^{(0)}\right|$ and the first-order correction to it, $\delta^{(0)}=\left|\psi_{g}^{(1)}-\psi_{g}^{(0)}\right|$, as functions of the momentum component $p_{x}$ and the angle $\theta$ between $\mathbf{k}_{1}$ and $\mathbf{k}_{2}$. The Cartesian coordinate system is introduced

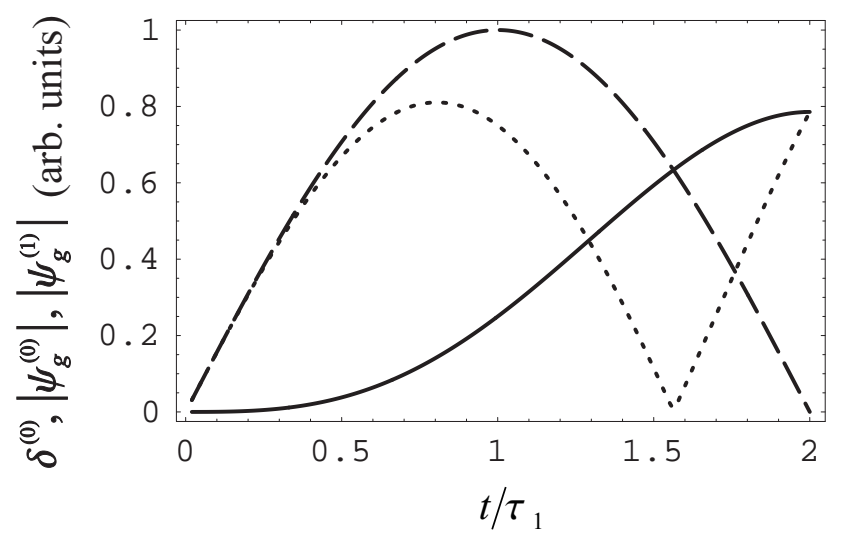

FIG. 4. Time dependences of $\delta^{(0)}$ (solid line), $\left|\psi_{g}^{(0)}\right|$ (long dashed line), and $\left|\psi_{g}^{(1)}\right|$ (short dashed line) for a two-mode object beam with $\mathbf{k}_{1,2} \perp \mathbf{k}_{0}$. The components of $\mathbf{p}$ are fixed as follows: $p_{y}=\hbar k_{0}$ and $p_{x}=p_{z}=0$. The coordinate axes and other parameters are the same as in Fig. 3.

in momentum space, $\mathbf{p}=\left(p_{x}, p_{y}, p_{z}\right)$, with the $x(y)$ axis directed along (opposite) the vector $\mathbf{k}_{0}\left(\mathbf{k}_{1}\right)$. Figure 3 shows the corresponding dependences after $\pi$-pulse time calculated for sodium atoms, provided that the initial wave packet has the Gaussian profile $\psi_{g}(\mathbf{p}) \propto$ $\exp \left[-L^{2}\left(\mathbf{p}-\mathbf{p}_{0}\right)^{2} /\left(2 \hbar^{2}\right)\right]$ with mean momentum $\mathbf{p}_{0}=$ $\hbar \mathbf{k}_{0},\left|\mathbf{k}_{0}\right|=k_{0}=1.07 \times 10^{5} \mathrm{~cm}^{-1}$, and spatial extension $2 L=0.4 \mathrm{~cm}$. The peaks in the central region of each plot correspond to forbidden values of $\theta \propto \delta_{k}<\delta_{p}\left(\tau_{1}\right)$. Outside these peaks $\left(|\theta|>10^{-4}\right)$ the relative correction goes down, approaching 0.15 at large $\theta$, that is, below its estimation value $\varepsilon_{r} \approx 0.5$.

In the worst case, e.g., $\mathbf{k}_{m} \perp \mathbf{k}_{0}, \forall m \geq 1$, the estimation for $\varepsilon_{r}$ is higher than $\delta_{p}\left(\tau_{1}\right) /\left(\hbar \delta_{k}\right)$, because it depends on $\delta_{\omega}=\hbar \delta_{k}^{2} /(2 M)$, the minimal kinetic energy an atom can get due to transition between the laser modes:

$$
\varepsilon_{r} \lesssim g_{\Sigma}\left|\int_{0}^{t} \sin ^{2}\left(g_{\Sigma} \tau\right) \exp \left(i \delta_{\omega} \tau\right) d \tau\right|
$$

Nevertheless, the term $\chi(\mathbf{p}, t)$ may still be treated as a small perturbation if the overall effective Rabi frequency satisfies the condition, more restrictive than Eq. (2.26),

$$
g_{\Sigma} \ll \delta_{\omega}
$$

Otherwise, if $g_{\Sigma} \gtrsim \delta_{\omega}$, the interaction time should be limited so that $t \ll \tau_{1}$. Figure 4 shows time dependences of $\delta^{(0)},\left|\psi_{g}^{(0)}\right|$, and $\left|\psi_{g}^{(1)}\right|$ for a two-mode object beam with $\delta_{p}\left(\tau_{1}\right) /\left(\hbar \delta_{k}\right)=0.1$ and $g_{\Sigma}=74 \delta_{\omega}$. We see that in the considered unfavorable configuration $\varepsilon_{r}$ does not exceed 0.1 even if $t=0.5 \tau_{1}$.

In most practical cases, however, only a small fraction of laser modes has a geometry leading to the condition (2.29), and the requirement (2.26) appears to be sufficient. 


\section{ATOM-OPTICS INTERPRETATION}

\section{A. General consideration}

In an idealized situation, one may imagine that all atoms are initially in a pure state described by the Gaussian profile

$$
g\left(\mathbf{p} ; \mathbf{p}_{0}\right)=\frac{L^{3 / 2}}{\hbar^{3 / 2} \pi^{3 / 4}} \exp \left(\frac{-L^{2}\left(\mathbf{p}-\mathbf{p}_{0}\right)^{2}}{2 \hbar^{2}}-\frac{i}{\hbar} \mathbf{p} \cdot \mathbf{r}_{0}\right)
$$

with mean momentum $\mathbf{p}_{0}$ close to $\hbar \mathbf{k}_{0}$, space position $\mathbf{r}_{0}$, and very small dispersion $\left[L \gg \hbar / \delta_{p}\left(\tau_{1}\right)\right]$. According to Eq. (2.7), after interaction with the laser beams over a time period $\tau \lesssim \tau_{1}$ and subsequent free propagation during time $t$ the atoms remain in a pure state, and their wave function can be represented as a superposition of useful signals $\psi^{(s, r)}\left(\mathbf{p}, \tau, t ; \mathbf{p}_{0}\right)$ and a background $\psi^{(b)}\left(\mathbf{p}, \tau, t ; \mathbf{p}_{0}\right)$, where

$$
\psi^{(\sigma)}\left(\mathbf{p}, \tau, t ; \mathbf{p}_{0}\right)=e^{-i w(\mathbf{p}) t} \int d \mathbf{p}^{\prime} G^{(\sigma)}\left(\mathbf{p}, \mathbf{p}^{\prime}, \tau\right) g\left(\mathbf{p}^{\prime} ; \mathbf{p}_{0}\right),
$$

$\sigma \in\{s, r, b\}$. The functions $G^{(\sigma)}\left(\mathbf{p}, \mathbf{p}^{\prime}, \tau\right)$ are defined by Eqs. (B2). In the case considered here they have explicit analytical expressions relying on the analogy with Eqs. (C1). These expressions are exact in the limit $L \rightarrow \infty$, and $\boldsymbol{\kappa} \rightarrow \mathbf{0}$, where $\boldsymbol{\kappa}=\mathbf{p}_{0} / \hbar-\mathbf{k}_{0}$. Omitting the irrelevant common phase factor $\exp \left[-i \mathbf{k}_{0} \cdot \mathbf{r}_{0}-i f_{0} \tau-\right.$ $\left.(i / \hbar) E_{|g\rangle}(0) \tau\right]$ we can readily infer that the Fourier transform of $\psi^{(r)}\left(\mathbf{p}, \tau, t ; \mathbf{p}_{0}\right)$,

$$
\psi_{r}\left(\mathbf{r}, \tau, t ; \mathbf{p}_{0}\right)=A_{r}(\hbar \boldsymbol{\kappa}, \tau) \gamma_{0}(\mathbf{r}, \boldsymbol{\kappa}) e^{i \mathbf{k}_{0} \cdot \mathbf{r}-i w\left(\hbar \mathbf{k}_{0}\right) t},
$$

propagates like the reference beam. Otherwise, the transform of $\psi^{(s)}\left(\mathbf{p}, \tau, t ; \mathbf{p}_{0}\right)$,

$$
\begin{aligned}
\psi_{s}\left(\mathbf{r}, \tau, t ; \mathbf{p}_{0}\right)= & A_{s}(\hbar \boldsymbol{\kappa}, \tau) \\
& \times \sum_{m \geq 1} \gamma_{m}(\mathbf{r}, \boldsymbol{\kappa}) \frac{g_{m}}{g_{\Sigma}} e^{i \mathbf{k}_{m} \cdot \mathbf{r}-i w\left(\hbar \mathbf{k}_{m}\right) t},
\end{aligned}
$$

generates a matter wave, which inherits the amplitude and phase characteristics of the object beam because $g_{m} \propto \mathbf{E}_{m}$ as follows from Eq. (A3c) and the definition of the Rabi frequencies $\Omega_{m}$. The last assertion also takes into account that all functions

$$
\gamma_{m}(\mathbf{r}, \boldsymbol{\kappa})=\frac{L^{3 / 2}}{\pi^{3 / 4} \sigma^{3}} \exp \left(-\frac{L^{2}\left(\mathbf{r}-\tilde{\mathbf{r}}_{t}^{m}\right)^{2}}{2|\sigma|^{4}}+i \delta_{\phi}\left(\mathbf{r}-\mathbf{r}_{t}^{m}\right)\right)
$$

used in Eqs. (3.3) and (3.4) slowly depend on $\mathbf{r}$ within spatial regions $\sim 2|\sigma|^{2} / L$, each centered around the point $\tilde{\mathbf{r}}_{t}^{m}=\mathbf{r}_{t}^{m}+\hbar(t+\tau) \boldsymbol{\kappa} / M$, where

$$
\sigma=\sqrt{L^{2}+i \hbar(t+\tau) / M}
$$

$$
\mathbf{r}_{t}^{m}=\mathbf{r}_{0}+\frac{\hbar\left(\mathbf{k}_{0}+\tilde{\mathbf{k}}\right)}{2 M} \tau+\frac{\hbar \mathbf{k}_{m}}{M} t
$$

and $\tilde{\mathbf{k}}$ is some typical wave vector in the object beam. The small phase shifts $\delta_{\phi}\left(\mathbf{r}-\mathbf{r}_{t}^{m}\right)$

$$
\delta_{\phi}(\mathbf{r})=\frac{1}{2|\sigma|^{4}}\left(L^{4} \mathbf{r} \cdot \boldsymbol{\kappa}+\frac{\hbar(t+\tau)\left(\mathbf{r}^{2}-L^{4} \boldsymbol{\kappa}^{2}\right)}{M}\right)
$$

introduced by $\gamma_{m}(\mathbf{r}, \boldsymbol{\kappa})$ vanish at small $\boldsymbol{\kappa}$ and large $L$.

If the overall effective Rabi frequency is chosen in agreement with the results of Sec. IID, the background, which itself represents a first-order correction to the wave function $\psi_{g}^{(0)}(\mathbf{p}, \tau)$, appears to be small at any time $\tau \lesssim \tau_{1}$. Consequently, since the states (3.3) and (3.4) are spatially separated after the free propagation period $t_{\text {min }}=2 L M /\left(\hbar \Delta_{k}\right)$, one may observe a matter wave $\psi_{s}\left(\mathbf{r}, \tau, t ; \mathbf{p}_{0}\right)$ cloning the object beam in the space-time region $\mathcal{S}=\left\{(\mathbf{r}, t):\left|\mathbf{r}-\tilde{\mathbf{r}}_{t}^{m}\right|<L, \forall m \geq 1 ; t>t_{\min }\right\}$, where all atomic wave packets related to different modes of this beam still overlap each other. It should be noted that $\mathcal{S} \neq \emptyset$ only when the observation time is limited by the value $t_{\max }=L M /\left[\hbar \tilde{k} \sin \left(\theta_{\max } / 2\right)\right]$, where $\theta_{\max }$ characterizes the maximal divergence angle of the object beam, and $\tilde{k}=|\tilde{\mathbf{k}}|$. In a given context, the physical meaning of conditions 2.26) and 2.29) consists in the requirement that a more delicate mechanism (lower laser intensity) has to be used in order to restore more detailed information. The conditions have a counterpart in the theory of optical holograms [see, e.g., Eq. (4) in Ref. [16]] which, in turn, is responsible for the low intensity of noise in the reconstructed wave.

In a more realistic case we may expect the initial atomic state to be a statistical mixture described by the density matrix

$$
\rho_{g g}\left(\mathbf{p}_{1}, \mathbf{p}_{2}, 0\right)=\int d \mathbf{p}^{\prime} f\left(\mathbf{p}^{\prime}\right) g\left(\mathbf{p}_{1} ; \mathbf{p}^{\prime}\right) g^{*}\left(\mathbf{p}_{2} ; \mathbf{p}^{\prime}\right),
$$

where $f(\mathbf{p})$ denotes a momentum distribution function. If this function is compatible with the condition (2.27), one can readily obtain an expression for $\rho_{g g}\left(\mathbf{p}_{1}, \mathbf{p}_{2}, t\right)$ at any time. In the region $\mathcal{S}$, it takes the following form in the coordinate representation

$\rho_{g g}\left(\mathbf{r}_{1}, \mathbf{r}_{2}, \tau, t\right)=\int d \mathbf{p}^{\prime} f\left(\mathbf{p}^{\prime}\right) \psi_{s}\left(\mathbf{r}_{1}, \tau, t ; \mathbf{p}^{\prime}\right) \psi_{s}^{*}\left(\mathbf{r}_{2}, \tau, t ; \mathbf{p}^{\prime}\right)$.

Since $A_{s}(\mathbf{p}, \tau)$ is a sharply peaked function having a width $\delta_{p}(\tau)$ along the vector $\boldsymbol{\Delta}_{k}$ [see Eq. (2.23)], the integral in Eq. (3.10) is limited in this direction. Let us assume that integration in the transverse directions is also restricted to a small domain $\sim \delta_{p}(\tau)$ due to the finite spectral width of $f(\mathbf{p})$. Then analyzing Eq. (3.10) 


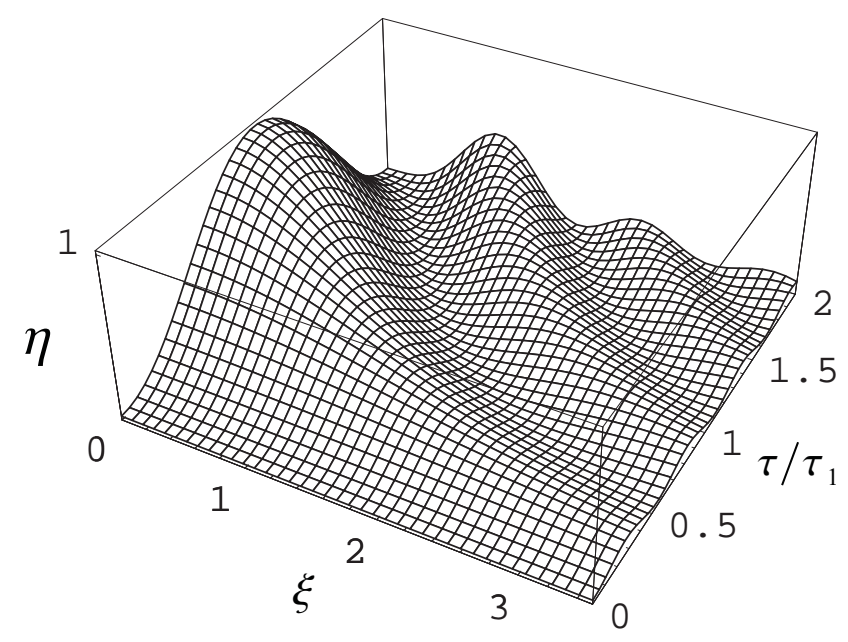

FIG. 5. Diffraction efficiency $\eta$ of atomic hologram as a function of time domain $\tau$ (in units of $\pi$ pulse) and dimensionless parameter $\xi$.

in the region $\left|\mathbf{r}_{1}-\mathbf{r}_{2}\right| \ll \hbar / \delta_{p}(\tau)$ one finds that under the condition $t \ll t_{\mathrm{coh}}=M /\left[\tilde{k} \sin \left(\theta_{\max } / 2\right) \delta_{p}(\tau)\right]$ the density matrix factorizes as a product of coherent states,

$$
\phi(\mathbf{r}, \tau, t)=C^{1 / 2}(\tau) \sum_{m \geq 1} \gamma_{m}(\mathbf{r}, \mathbf{0}) \frac{g_{m}}{g_{\Sigma}} e^{i \mathbf{k}_{m} \cdot \mathbf{r}-i w\left(\hbar \mathbf{k}_{m}\right) t},
$$

where

$$
C(\tau)=\int d \mathbf{p}\left|A_{s}(\mathbf{p}, \tau)\right|^{2} f\left(\mathbf{p}+\hbar \mathbf{k}_{0}\right) .
$$

When deriving the formula (3.11) we allow for small values of phase differences $\left|\delta_{\phi}\left(\mathbf{r}_{1}-\mathbf{r}_{t}^{m}\right)-\delta_{\phi}\left(\mathbf{r}_{2}-\mathbf{r}_{t}^{n}\right)\right| \ll$ $\pi, \forall m, n \geq 1$, appearing in the integration region at $t \ll t_{\text {coh }}$, whence $\gamma_{m}(\mathbf{r}, \boldsymbol{\kappa}) \simeq \gamma_{m}(\mathbf{r}, \mathbf{0})$. Note that mutual compatibility of time conditions $t_{\min }<t \ll t_{\text {coh }}$ restricts the possible structure of the object beam:

$$
\sin \left(\theta_{\max } / 2\right) \ll \frac{\hbar \Delta_{k}}{2 L \tilde{k} \delta_{p}(\tau)} .
$$

We see that the superposition of laser beams selectively acts only on those wave packets in the initial representation of the atomic density matrix Eq. (3.9) whose spectra are concentrated near the vector $\hbar \mathbf{k}_{0}$, and thus restores the pure state Eq. (3.11). Therefore the inhomogeneous laser radiation proves to behave like a three-dimensional hologram with respect to the incident atomic beam (impinging wave packets).

One can further establish a close relation between an atomic hologram created in a time domain $\tau$ and a permanent optical hologram with the thickness $d_{\tau}=$ $\hbar k_{0} \tau / M$ along the direction of the reading beam. Indeed, as is known from optics, the passage of the reading beam through a three-dimensional hologram can be interpreted as a multiple diffraction in which small waves, diffracted from different registration-medium layers with equivalent transmission of light, interfere constructively to form a reconstructed wave of high intensity. The same approach can be used to describe an atom-optics hologram as a light structure, inducing an optical potential through the atom-laser dipole interaction [12]. Here the role of equivalent-transmission layers in the medium is performed by the equipotential surfaces. Since $d_{\tau}$ is just the distance the impinging wave packet covers during time $\tau$, the numbers of crossed interfaces (layers or surfaces) are equal for the atomic and conventional holograms. Therefore, if there were no difference in the initial and boundary conditions, the processes of wave front reconstruction would be identical in both cases.

The relation between atomic and optical holograms makes it possible to classify atomic holograms as thin or thick diffractive optical elements, and use the Talbot length $L_{\text {Talbot }}$, i.e., the typical interval between consecutive interfaces, as a characteristic scale to distinguish between the two classes 17. That is, the hologram can be considered as thick (three dimensional) if $d_{\tau}>L_{\text {Talbot }}$, or in terms of time

$$
\tau>L_{\text {Talbot }} M /\left(\hbar k_{0}\right) .
$$

For most holographic setups (for instance, like that in Fig. 2) $L_{\text {Talbot }} \sim 2 \pi / k_{0}$. Therefore the criterion (3.14) leads to a time domain $\tau$ larger than the period of atomic oscillations. Obviously the latter requirement is well satisfied for $\tau \sim \tau_{1}$, the time of the $\pi$ pulse, provided $g_{\Sigma}$ is chosen in agreement with the condition (2.26).

\section{B. Diffraction efficiency}

In a regime where the background is small, we can define the diffraction efficiency $\eta$ of a hologram as the overall intensity of the modes composing the reconstructed wave, provided the initial wave packet is normalized to 1 ,

$$
\eta\left(\tau, \mathbf{p}_{0}\right)=\int d \mathbf{p}\left|\psi^{(s)}\left(\mathbf{p}, \tau, t ; \mathbf{p}_{0}\right)\right|^{2} .
$$

It is clear, however, that $\eta$ depends on the shape of the initial distribution as well. Therefore, to be more specific let us assume a Gaussian profile (3.1) of the impinging wave packet with infinitely small dispersion $L \rightarrow \infty$. Then integration over $\mathbf{p}^{\prime}$ in Eq. (3.2) becomes trivial, so that

$$
\eta\left(\tau, \mathbf{p}_{0}\right)=\int d \mathbf{p}\left|G^{(s)}\left(\mathbf{p}, \mathbf{p}_{0}, \tau\right)\right|^{2} .
$$

Using the approximate expressions (C1b) and omitting negligible interference terms one obtains from the above equation

$$
\eta\left(\tau, \mathbf{p}_{0}\right)=\eta(\tau, \xi) \simeq \frac{1}{\xi^{2}+1} \sin ^{2}\left(\tau g_{\Sigma} \sqrt{\xi^{2}+1}\right),
$$


where the dimensionless parameter

$$
\xi=\frac{\left(\mathbf{p}_{0}-\hbar \mathbf{k}_{0}\right) \cdot \boldsymbol{\Delta}_{k}}{M g_{\Sigma}}
$$

characterizes the deviation of the initial atomic momentum from the mean momentum of photons in the reference beam.

According to this simple formula the diffraction efficiency achieves a maximum at $\tau=\tau_{n} / \sqrt{\xi^{2}+1}$ and can reach $100 \%$ if $\xi=0$ (see Fig. 5).

\section{Numerical example}

In the following we show two-dimensional results obtained for $\mathrm{Na}$ assuming an experimental setup like that in Fig. 2 (i.e., all $\mathbf{k}_{m}$ are coplanar vectors and $\tilde{\mathbf{k}} \perp \mathbf{k}_{0}$ ). The image to be reconstructed is a thin line of width $\lambda=2 \pi / k_{0}$ perpendicular to the laser-beam plane. To decrease the amount of computational work we reduced the number of object wave modes to 31 and set up $\theta_{\max }=\pi / 4$. Such a field approaches the desired single line within a region of size $\sim 60 \lambda$ centered around the point $\mathbf{r}=\mathbf{0}$ if all the laser modes in the expression (2.5) have identical amplitudes $\mathbf{E}_{m}$, and their wave vectors $\mathbf{k}_{m}$ are equidistant

$$
\mathbf{k}_{m}=k_{0}\left(\sin \left[\frac{\pi(m-16)}{120}\right],-\cos \left[\frac{\pi(m-16)}{120}\right], 0\right) .
$$

The corresponding profile of the object beam intensity distribution $I(x)$ is shown in Fig. 6, where the $x$ axis is directed along the vector $\mathbf{k}_{0}=\left(k_{0}, 0,0\right)$. The interference fringes, which are a consequence of the finite number of modes, can easily be separated from the central line and therefore do not contaminate our consideration.

In numerical simulations the optical pulse duration was taken to be $\tau_{1}=2.82 \times 10^{-2} \mathrm{~s}$, to demonstrate the highest diffraction efficiency. The remaining laser light parameters were fixed as follows: Rabi frequencies $\Omega_{0}=1$ $\mathrm{MHz}$ and $\Omega_{m}=0.01 \Omega_{0}$ for all $1 \leq m \leq 31$, detuning $\Delta=-1 \mathrm{GHz}(\gamma / \Delta \approx 0.06)$, the effective Rabi frequencies $g_{m}=10 \mathrm{~Hz}, f_{m n}=0.1 \mathrm{~Hz}$, and $g_{\Sigma}=55.7$ Hz. Note that for the considered laser-beam geometry $\Delta_{k}=\sqrt{2} k_{0}=1.51 \times 10^{5} \mathrm{~cm}^{-1}$ and $\delta_{k}=3.28 \times 10^{3}$ $\mathrm{cm}^{-1}$, so that the background introduces a relative correction of the order $\varepsilon_{r}=\delta_{p}\left(\tau_{1}\right) /\left(\hbar \delta_{k}\right)=1.4 \times 10^{-2}$ and can be neglected.

The reconstruction of a real image of the object was achieved by impinging Gaussian wave packets (3.1) having spatial extension $2 L=0.4 \mathrm{~cm}$ on the superposition of laser beams near the point

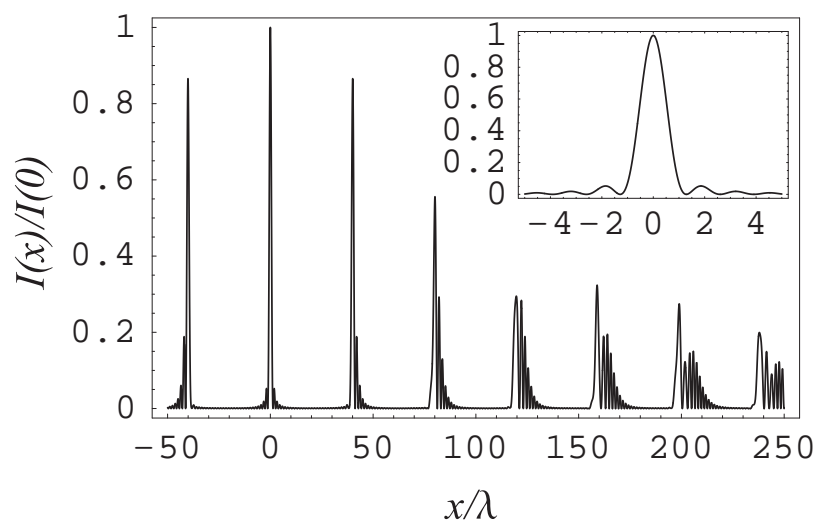

FIG. 6. Intensity $I(x)$ of the 31-mode object wave as a function of observation point $\mathbf{r}=(x, 0,0)$. The inset shows optical image of a single line $\sim \lambda$ created in the central region $\sim 60 \lambda$.

$$
\mathbf{r}_{0}=\left(-\frac{\hbar k_{0} \tau_{1}}{M}, \frac{L}{\tan \left(\theta_{\max } / 2\right)}-\frac{\hbar k_{0} \tau_{1}}{M}, 0\right) .
$$

After the interaction with laser radiation is over, these wave packets appear at a distance $L / \tan \left(\theta_{\max } / 2\right)=0.48$ $\mathrm{cm}$ from the image. As a result, the most intensive matter field in the imaging region may be observed after free propagation time $t=t_{\max } \cos \left(\theta_{\max } / 2\right)=0.16 \mathrm{~s}$, which lies within the limits $t_{\min }=9.6 \times 10^{-2} \mathrm{~s}$ and $t_{\max }=0.17$ s. Figure 7 shows the corresponding atomic density profile $\rho_{g g}\left(\mathbf{r}, \mathbf{r}, \tau_{1}, t\right)$ when the mean momentum of the initial wave packet is exactly equal to $\hbar \mathbf{k}_{0}$. As is seen from the bottom part of the plot, the atomic profile displays a good match with the distribution of the object beam intensity. The attained diffraction efficiency calculated according to Eq. (3.15) is $98 \%$ in this case.

When initial state is a statistical mixture (3.9) with momentum distribution function $f(\mathbf{p})$ uniform along the $x$ axis, the atomic density profile acquires a shape represented in the Fig. 8. Since condition (3.13) does not hold at the chosen laser light parameters, the size of the reconstructed line appears to be $\sim 4$ times wider than one might expect from a coherent reading beam. Nevertheless, such image broadening is not too large, so that the atomic hologram can be used even in this unfavorable design.

\section{CONCLUSIONS}

In this paper we have studied a method of driving ultracold atom propagation using effective holograms made of laser radiation in a specified time domain. We have shown that the scattered atomic wave packet may inherit the features of the object electromagnetic wave provided the atomic internal ground state possesses a translation invariance due to compensation of gravity with the SternGerlach effect. We have established a close relation 


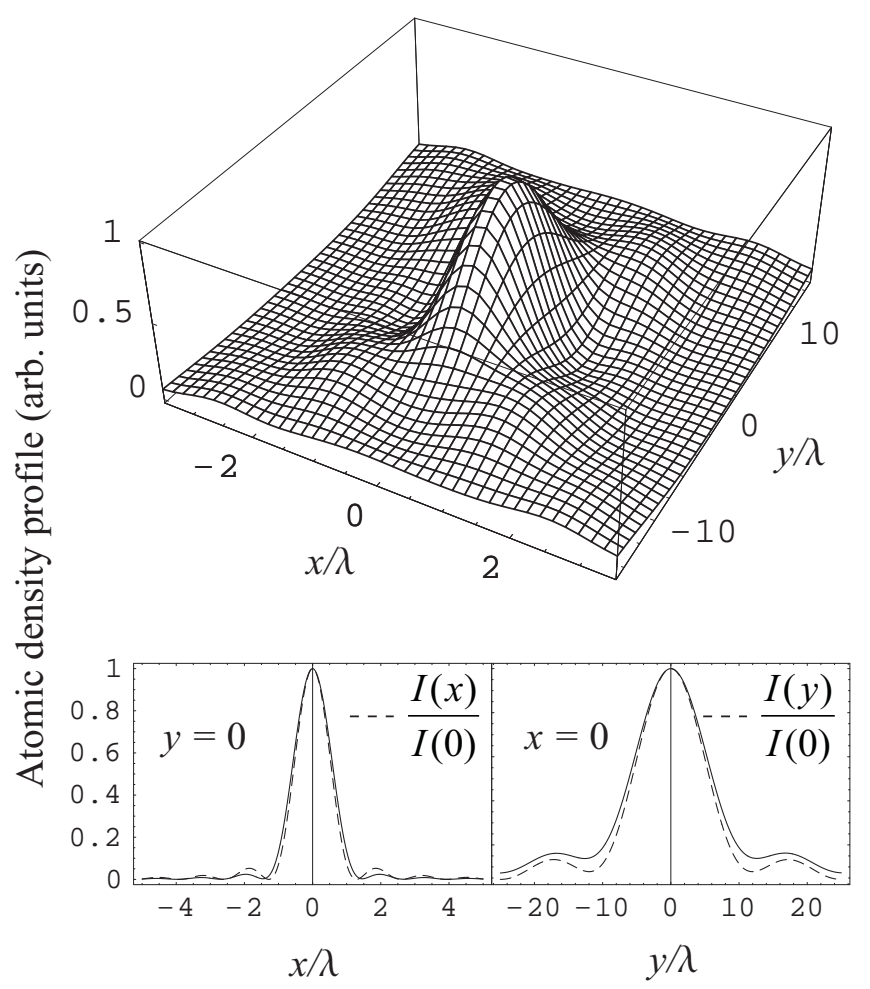

FIG. 7. Atomic density $\rho_{g g}\left(\mathbf{r}, \mathbf{r}, \tau_{1}, t\right)$ as a function of observation point $\mathbf{r}=(x, y, 0)$. The bottom part of the plot compares atomic profile (solid lines) with the object beam intensity distributions $I(x)$ and $I(y)$ (dashed lines) in the planes $y=0$ and $x=0$.

between the atomic hologram created in the time domain and a thick optical hologram prepared in the corresponding spatial region, and have found a recipe for controlling the diffraction efficiency of such an atomic hologram by means of varying the time domain. Besides adjusting the atom-laser interaction time, another way to enhance diffraction efficiency has proved to be the cooling of the atomic beam so that all the particles get the same momentum as the momentum of photons in the reference wave. A special role here may be played by BEC and coherent atomic-beam generators, which are under development now [23].

We have considered dilute atomic samples, i.e., we have not included many-atom interactions 24, which may lead to nonlinear atom-optics effects [25] along with enhancing the background. The conditions under which these interactions can be neglected were elaborated in our previous paper 12 using the mean-field approximation applied to the Maxwell-Bloch equations [26] and are well satisfied when the mean-field interaction energy per particle is much less than the typical kinetic energy of an atom. We have also neglected such possible sources of background as spontaneous emission of photons and fluctuations of the laser frequency. While the first of these sources may be eliminated by keeping the laser

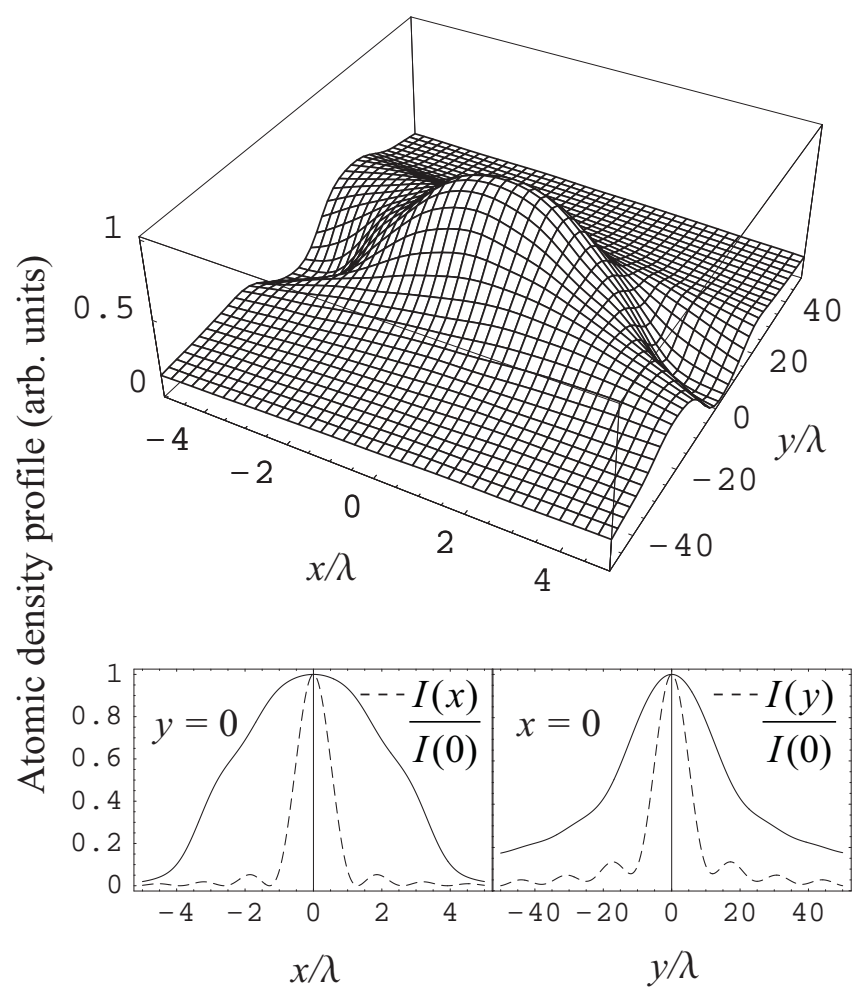

FIG. 8. Atomic density $\rho_{g g}\left(\mathbf{r}, \mathbf{r}, \tau_{1}, t\right)$ obtained when the initial state is a statistical mixture with uniform momentum distribution along the $x$ axis. Other notations are the same as in Fig. 1.

detuning much bigger than the spontaneous emission rate, the second one is determined by the spectral width of two-time electromagnetic-field correlation functions 12, 27, and substantially decreases if all field modes originate from one initial laser mode.

Although our scheme of an atomic hologram has been developed for codirected reading and reference beams it can readily be modified for an experimental setup with opposite propagation of the beams. In full analogy with conventional optics, such a hologram will reconstruct the conjugate object wave.

In conclusion, atom-optics holograms appear to be a useful implement for solving some of the basic technological problems in the field of atom lithography. For instance, they will make it possible to grow $3 \mathrm{D}$ circuitry components by depositing an arbitrary multilayer pattern of impurity atoms on a silicon substrate.

\section{APPENDIX A: ELIMINATION OF THE EXCITED STATE}

In the rotating wave approximation the twocomponent Schrödinger equation, rewritten for groundand excited-level wave functions $\psi_{g}(\mathbf{p}, t)$ and $\psi_{e}(\mathbf{p}, t)$ slowly varying in time, takes the form 


$$
\begin{aligned}
i \frac{\partial}{\partial t} \psi_{g}(\mathbf{p}, t)= & {[w(\mathbf{p})+\Delta] \psi_{g}(\mathbf{p}, t) } \\
& -\sum_{m \geq 0} \Omega_{m}^{*} \psi_{e}\left(\mathbf{p}+\hbar \mathbf{k}_{m}, t\right), \\
i \frac{\partial}{\partial t} \psi_{e}(\mathbf{p}, t)= & {\left[w(\mathbf{p})-i \mathbf{f}_{e} \cdot \nabla\right] \psi_{e}(\mathbf{p}, t) } \\
& -\sum_{m \geq 0} \Omega_{m} \psi_{g}\left(\mathbf{p}-\hbar \mathbf{k}_{m}, t\right),
\end{aligned}
$$

where $\Omega_{m}$ stands for the Rabi frequency of mode $m$ and the terms $w(\mathbf{p})=\mathbf{p}^{2} /(2 M \hbar)$ and $-i \mathbf{f}_{e} \cdot \nabla$ arise in momentum space from the kinetic and potential energy $\left(-\mathbf{f}_{e} \cdot \mathbf{r}\right)$ respectively.

The route by which one can adiabatically eliminate the component $\psi_{e}(\mathbf{p}, t)$ from Eqs. (A1) implies a selfconsistent assumption $\left|\psi_{e}\right| \ll\left|\psi_{g}\right|$ leading to the zeroorder solution of Eq. (A10): $\psi_{g}(\mathbf{p}, t) \simeq \exp \{-i[w(\mathbf{p})+$ $\Delta] t\} \psi_{g}(\mathbf{p}, t=0)$. After substitution of this expression into Eq. (A1b) the latter is solved perturbatively with respect to the potential energy term:

$$
\psi_{e}(\mathbf{p}, t) \simeq-\sum_{m \geq 0} \frac{\Omega_{m} \psi_{g}\left(\mathbf{p}-\hbar \mathbf{k}_{m}, t\right)}{w(\mathbf{p})-w\left(\mathbf{p}-\hbar \mathbf{k}_{m}\right)-\Delta}+\ldots,
$$

where the ellipsis denotes omitted terms that include a small $\left(\propto\left|\mathbf{f}_{e}\right| L /|\hbar \Delta|\right)$ first-order correction to $\psi_{e}(\mathbf{p}, t)$ and also terms that oscillate with the nonresonant frequency $w(\mathbf{p})$ and therefore give a negligible contribution when one uses the above expression within the context of Eq. (A1a).

For an ultracold atomic sample one can further discard the kinetic energy terms in the denominators of the expression (A2). As a result, Eq. (A1a) takes the form of Eq. (2.9), provided that the effective Rabi frequencies $f_{0}$, $f_{m n}$, and $g_{m}$ are defined as follows:

$$
\begin{gathered}
f_{0}=\frac{1}{\Delta} \sum_{m \geq 0}\left|\Omega_{m}\right|^{2}, \\
f_{m n}=\frac{\Omega_{m} \Omega_{n}^{*}}{\Delta}, \\
g_{m}=\frac{\Omega_{m} \Omega_{0}^{*}}{\Delta} .
\end{gathered}
$$

\section{APPENDIX B: GROUND-STATE GREEN FUNCTION}

Here we present the first-order approximation to the ground-state component of the Green function determining the time evolution of the atomic density matrix according to formula (2.7):

$$
G_{g g}\left(\mathbf{p}, \mathbf{p}^{\prime}, t\right)=e(t) \sum_{\sigma \in\{r, s, b\}} G^{(\sigma)}\left(\mathbf{p}, \mathbf{p}^{\prime}, t\right),
$$

where common phase multiplier $e(t)=\exp [i \omega t-$ $\left.(i / \hbar) E_{|e\rangle}(0) t\right]$ recovers the solution (2.21) from its slow time dependence, and

$$
\begin{gathered}
G^{(r)}\left(\mathbf{p}, \mathbf{p}^{\prime}, t\right)=\mathcal{M}\left[\phi_{0}^{(0)}\left(\mathbf{p}, \mathbf{p}^{\prime}, \lambda\right)\right], \\
G^{(s)}\left(\mathbf{p}, \mathbf{p}^{\prime}, t\right)=\sum_{m \geq 1} \mathcal{M}\left[\phi_{m}^{(0)}\left(\mathbf{p}, \mathbf{p}^{\prime}, \lambda\right)\right], \\
G^{(b)}\left(\mathbf{p}, \mathbf{p}^{\prime}, t\right)=\sum_{m \geq 0} \mathcal{M}\left[\phi_{m}^{(b)}\left(\mathbf{p}, \mathbf{p}^{\prime}, \lambda\right)\right] .
\end{gathered}
$$

In these formulas the operator $\mathcal{M}$ stands for the inverse Laplace transformation and shift of the momentum arguments

$$
\begin{aligned}
\mathcal{M}\left[\phi_{m}^{(\sigma)}\left(\mathbf{p}, \mathbf{p}^{\prime}, \lambda\right)\right] \equiv & 2 \pi i \int_{\epsilon-i \infty}^{\epsilon+i \infty} d \lambda e^{\lambda t} \\
& \times \phi_{m}^{(\sigma)}\left(\mathbf{p}-\hbar \mathbf{k}_{m}, \mathbf{p}^{\prime}, \lambda\right),
\end{aligned}
$$

$\epsilon>0, \sigma \in\{0, b\}, m \geq 0$, whereas

$$
\begin{gathered}
\phi_{0}^{(0)}\left(\mathbf{p}, \mathbf{p}^{\prime}, \lambda\right)=\frac{-i}{T(\mathbf{p}, \lambda)} \delta^{3}\left(\mathbf{p}+\hbar \mathbf{k}_{0}-\mathbf{p}^{\prime}\right), \\
\phi_{0}^{(b)}\left(\mathbf{p}, \mathbf{p}^{\prime}, \lambda\right)=\frac{i}{T(\mathbf{p}, \lambda)^{2}} \chi\left(\mathbf{p}, \mathbf{p}^{\prime}, \lambda\right), \\
\phi_{m}^{(\sigma)}\left(\mathbf{p}, \mathbf{p}^{\prime}, \lambda\right)=\frac{-g_{m}}{w_{m}(\mathbf{p})-i \lambda} \phi_{0}^{(\sigma)}\left(\mathbf{p}, \mathbf{p}^{\prime}, \lambda\right),
\end{gathered}
$$

$m \geq 1$, and the expression for $\chi\left(\mathbf{p}, \mathbf{p}^{\prime}, \lambda\right)$ is obtained from Eq. (2.16),

$$
\begin{aligned}
\chi\left(\mathbf{p}, \mathbf{p}^{\prime}, \lambda\right)= & \sum_{m \geq 1}\left(\sum_{\substack{n \geq 0 \\
n \neq m}} g_{m}^{*} \phi_{n}^{(b)}\left[\mathbf{p}+\hbar\left(\mathbf{k}_{m}-\mathbf{k}_{n}\right), \mathbf{p}^{\prime}, \lambda\right]\right. \\
& +\sum_{n \geq 1} g_{n} \phi_{m}^{(b)}\left[\mathbf{p}+\hbar\left(2 \mathbf{k}_{0}-\mathbf{k}_{n}-\mathbf{k}_{m}\right), \mathbf{p}^{\prime}, \lambda\right] \\
& +\sum_{\substack{n \geq 1 \\
n \neq m}} \sum_{l \geq 0} f_{m n} \\
& \left.\times \phi_{l}^{(b)}\left[\mathbf{p}+\hbar\left(\mathbf{k}_{0}-\mathbf{k}_{l}-\mathbf{k}_{m}+\mathbf{k}_{n}\right), \mathbf{p}^{\prime}, \lambda\right]\right) .
\end{aligned}
$$




\section{APPENDIX C: NEAR-RESONANCE APPROXIMATION}

Here we develop the near-resonance approximation in order to get an explicit solution of the truncated system of equations (2.13) and (2.15). In the region $\mathcal{D}$ one can approximately treat the kinetic energy terms $w_{m}(\mathbf{p})$ corresponding to the different modes of the object beam $(m \geq 1)$ as equal: $w_{m}(\mathbf{p}) \approx w_{n}(\mathbf{p}) \approx \tilde{w}(\mathbf{p})$, where $\tilde{w}(\mathbf{p})=w(\mathbf{p}+\hbar \tilde{\mathbf{k}})+\Delta+f_{0}$, and $\tilde{\mathbf{k}}$ is some typical wave vector in the object beam. Under this condition the integral in Eq. 2.21) can be calculated explicitly, and the wave functions $\psi_{m}^{(0)}(\mathbf{p}, t)$ acquire a simple analytical representation,

$$
\begin{gathered}
\psi_{0}^{(0)}(\mathbf{p}, t) \simeq A_{r}(\mathbf{p}, t) e^{-i b(\mathbf{p}) t} \psi_{g}\left(\mathbf{p}+\hbar \mathbf{k}_{0}\right), \\
\psi_{m}^{(0)}(\mathbf{p}, t) \simeq A_{s}(\mathbf{p}, t) \frac{g_{m}}{g_{\Sigma}} e^{-i b(\mathbf{p}) t} \psi_{g}\left(\mathbf{p}+\hbar \mathbf{k}_{0}\right), \quad m \geq 1
\end{gathered}
$$

In these formulas,

$$
\begin{gathered}
A_{r}(\mathbf{p}, t)=\frac{i a(\mathbf{p})}{d(\mathbf{p})} \sin [d(\mathbf{p}) t]+\cos [d(\mathbf{p}) t], \\
A_{s}(\mathbf{p}, t)=\frac{-i g_{\Sigma}}{d(\mathbf{p})} \sin [d(\mathbf{p}) t],
\end{gathered}
$$

where

$$
\begin{gathered}
a(\mathbf{p})=\left[\tilde{w}(\mathbf{p})-w_{0}(\mathbf{p})\right] / 2 \\
b(\mathbf{p})=a(\mathbf{p})+w_{0}(\mathbf{p}) \\
d(\mathbf{p})=\sqrt{a(\mathbf{p})^{2}+g_{\Sigma}^{2}}
\end{gathered}
$$

and

$$
g_{\Sigma}=\left(\sum_{m \geq 1}\left|g_{m}\right|^{2}\right)^{1 / 2}
$$

stands for the overall effective Rabi frequency.

[1] A. Aspect, E. Arimondo, R. Kaiser, N. Vansteenkiste, and C. Cohen-Tannoudji, Phys. Rev. Lett. 61, 826 (1988); J. Lawall, S. Kulin, B. Saubamea, N. Bigelow, M. Leduc, and C. Cohen-Tannoudji, ibid. 75, 4194 (1995).
[2] M. Kasevich and S. Chu, Phys. Rev. Lett. 69, 1741 (1992); N. Davidson, H. J. Lee, M. Kasevich, and S. Chu, ibid. 72, 3158 (1994); H. J. Lee, C. S. Adams, M. Kasevich, and S. Chu, ibid. 76, 2658 (1996).

[3] V. I. Balykin, V. S. Letokhov, Yu. B. Ovchinnikov, and A. I. Sidorov, Pis'ma Zh. Eksp. Teor. Fiz. 45, 282 (1987) [JETP Lett. 45, 353 (1987)]; M. Arndt, P. Szriftgiser, J. Dalibard, and A. M. Steane, Phys. Rev. A 53, 3369 (1996); N. Friedman, R. Ozeri, and N. Davidson, J. Opt. Soc. Am. B 16, 1749 (1998).

[4] P. E. Moskowitz, P. L. Gould, S. R. Atlas, and D. E. Pritchard, Phys. Rev. Lett. 51, 370 (1983).

[5] P. J. Martin, B. G. Oldaker, A. N. Miklich, and D. E. Pritchard, Phys. Rev. Lett. 60, 515 (1988).

[6] V. I. Balykin, I. I. Klimov, and V. S. Letokhov, Pis'ma Zh. Eksp. Teor. Fiz. 59, 219 (1994) [JETP Lett. 59, 235 (1994)]; M. K. Olsen, T. Wong, S. M. Tan, and D. F. Walls, Phys. Rev. A 53, 3358 (1996).

[7] D. Gabor, Nature (London) 161, 777 (1948); Proc. R. Soc. London, Ser. A 197, 454 (1949).

[8] G. Timp, R. E. Behringer, D. M. Tennant, J. E. Cunningham, M. Prentiss, and K. K. Berggren, Phys. Rev. Lett. 69, 1636 (1992); J. J. McClelland, R. E. Scholten, E. C. Palm, and R. J. Celotta, Science 262, 877 (1993); R. Gupta, J. J. McClelland, Z. J. Jabbour, and R. J. Celotta, Appl. Phys. Lett. 67, 1378 (1995).

[9] M. Moringa, M. Yasuda, T. Kishimoto, F. Shimizu, J.T. Fujita, and S. Matsui, Phys. Rev. Lett. 77, 802 (1996).

[10] O. Zobay, E. V. Goldstein, and P. Meystre, Phys. Rev. A 60, 3999 (1999).

[11] M. Anderson, J. R. Ensher, M. R. Matthews, C. E. Wieman, and E. A. Cornell, Science 269, 198 (1995); K. B. Davis, M.-O. Mewes, M. R. Andrews, N. J. van Druten, D. S. Durfee, D. M. Kurn, and W. Ketterle, Phys. Rev. Lett. 75, 3969 (1995); C. C. Bradley, C. A. Sackett, J. J. Tollett, and R. Hulet, ibid. 75, 1687 (1995).

[12] A. V. Soroko, J. Phys. B. 30, 5621 (1997).

[13] M. Olshanii, N. Dekker, C. Herzog, and M. Prentiss, eprint quant-ph/9811021.

[14] H. Kogelnik, Bell Syst. Techn. J. 48, 2909 (1969).

[15] P. P. Ewald, Ann. Phys. (Leipzig) 54, 519 (1917).

[16] V. G. Sidorovich, Zh. Tekh. Fiz. 46, 1306 (1976) [Sov. Phys. Tech. Phys. 21, 742 (1976)].

[17] M. K. Oberthaler, R. Abfalterer, S. Bernet, C. Keller, J. Schmiedmayer, and A. Zeilinger, Phys. Rev. A 60, 456 (1999).

[18] A. V. Soroko, Phys. Rev. A 58, 3963 (1998).

[19] M.-O. Mewes, M. R. Andrews, D. M. Kurn, D. S. Durfee, C. G. Townsend, and W. Ketterle, Phys. Rev. Lett. 78, 582 (1997).

[20] K. Moler, D. S. Weiss, M. Kasevich, and S. Chu, Phys. Rev. A 45, 342 (1992).

[21] E. A. Korsunsky, D. V. Kosachiov, B. G. Matisov, and Yu. V. Rozhdestvensky, Zh. Eksp. Teor. Fiz. 103, 396 (1993) [JETP 76, 210 (1993)].

[22] A. P. Kazantsev, G. A. Ryabenko, G. I. Surdutovich, and V. P. Yakovlev, Phys. Rep. 129, 75 (1985).

[23] R. J. C. Spreeuw, T. Pfau, U. Janicke, and M. Wilkens, Europhys. Lett. 32, 469 (1995); H. M. Wiseman and M. J. Collett, Phys. Lett. A 202, 246 (1995); M. Holland, 
K. Burnett, C. Gardiner, J. I. Cirac, and P. Zoller, Phys. Rev. A 54, R1757 (1994); A. M. Guzman, M. Moore, and P. Meystre, ibid. 53, 977 (1996); G. M. Moy, J. J. Hope, and C. M. Savage, ibid. 55, 3631 (1997).

[24] M. Lewenstein, L. You, J. Cooper, and K. Burnett, Phys. Rev. A 50, 2207 (1994).

[25] W. Zhang and D. F. Walls, Phys. Rev. A 49, 3799 (1994); G. Lenz, P. Meystre, and E. M. Wright, ibid. 50, 1681 (1994).

[26] Y. Castin and K. Mølmer, Phys. Rev. A 51, R3426 (1995).

[27] B. J. Dalton and P. L. Knight, J. Phys. B. 15, 3997 (1982). 